\title{
Correlation of Anxiety Levels between Temporomandibular Disorder Patients and Normal Subjects
}

\author{
Subhash Vasudeva, ${ }^{1}$ Asha Iyengar, ${ }^{1}$ and Nagesh Seetaramaiah ${ }^{2}$ \\ ${ }^{1}$ Department of Oral Medicine and Radiology, DAPM RV Dental College, CA 37, 24th Main, J P Nagar 1st Phase, \\ Bangalore 560078, India \\ ${ }^{2}$ Department of Public Health and Centre for Disease Control, Rajiv Gandhi University of Health Sciences, Bangalore, India
}

Correspondence should be addressed to Subhash Vasudeva; subash75adiga@gmail.com

Received 30 April 2014; Accepted 2 September 2014; Published 14 September 2014

Academic Editor: Mitsuhiro Ohshima

\begin{abstract}
Copyright (C) 2014 Subhash Vasudeva et al. This is an open access article distributed under the Creative Commons Attribution License, which permits unrestricted use, distribution, and reproduction in any medium, provided the original work is properly cited.

Background. Temporomandibular disorders (TMDs) are among the common musculoskeletal conditions affecting the individual. Anxiety plays an important role in the pathogenesis of TMD. Modern lifestyle and work environment bring to focus the role of anxiety in everyday life which is changing the demographics of diseases like TMD. This study compared the anxiety scores between TMD patients and normal subjects. Material and Methods. 505 individuals were included in the study who were divided into group 1 with 255 individuals presenting with signs and symptoms of TMD and group 2 with 250 normal individuals as controls. Hospital anxiety depression scale was used to calculate the anxiety scores. Fischer's $t$ test was used to compare the anxiety scores between the two groups. Results. $80 \%$ of individuals in group 2 and $44 \%$ in group 1 individuals had normal anxiety scores. $45 \%$ of the individuals in group 1 and $19 \%$ in group 2 had borderline anxiety scores. $11 \%$ of group 1 individuals and $<1 \%$ of group 2 individuals demonstrated high anxiety scores. These results were statistically significant. Conclusion. Individuals with TMD exhibited higher levels of anxiety scores whereas significant number of control subjects exhibited normal anxiety scores.
\end{abstract}

\section{Introduction}

Temporomandibular disorders (TMDs) are now recognized as a group of biopsychosocial illnesses characterized by chronic painful conditions and dysfunctions in the muscles of mastication and temporomandibular joint (TMJ) [1]. TMDs affect $5-10 \%$ of the population and it is estimated to incur $\$ 1$ billion in health care costs [2].

The etiopathogenesis of TMD is complex with many risk factors including trauma to the TMJ area, anatomical factors, psychosocial profile, and sensitization of pain carrying neural pathways playing a role in causation of TMD [2]. Genetic basis for the development of TMD has been suggested by studies involving twins and family members $[3,4]$. Individuals with TMD may present with TM joint pain, headache, ear ache, clicking and popping sounds, and alteration in jaw movements [5]. Apart from orofacial pain and alteration in jaw mechanics, TMD patients demonstrate enhanced pain sensitivity and psychological dysfunction due to impairment in CNS mediated regulatory processes [5]. Furthermore, physical and emotional stress along with altered adrenergic receptor mediated responses due to gene polymorphisms can increase the chances of developing TMD [6]. Modern lifestyle and work culture bring stress into main focus. Individuals who are always on the run, unknowingly, are entering the world of stress and its harmful effects. Due to this, the demographics of various disease processes including TMD is changing. Psychological factors are found to influence the treatment outcomes in majority of painful TMD patients. Studies in different clinical settings have shown an association between anxiety, depression, and TMD [7-10]. This has led to a multidisciplinary approach including the psychological component in the management of TMD [1]. Taking these into consideration a study was attempted to analyze the role of anxiety and depression in TMDs and investigate the prevalence rates of anxiety in individuals affected with TMD 
in comparison with normal individuals in a small subset of Bangalore (India) city population.

\section{Material and Methods}

Ethical clearance from the institutional review board was obtained for conducting the study. 505 subjects aged between 18 and 49 years were selected for the study. The subjects were divided into 2 groups. Group 1 is comprised of 255 subjects with signs and symptoms of TMD and group 2 is comprised of 250 asymptomatic subjects without any signs and symptoms of TMD. The asymptomatic subjects acted as controls.

Inclusion criteria for control group include the following:

(i) subjects without any signs and symptoms of TMD

(ii) subjects who are not suffering from systemic conditions which may affect the functioning of TMJ, for example, rheumatoid arthritis, scleroderma, and septic arthritis;

(iii) subjects without any comorbid conditions like hypertension, diabetes, asthma, epilepsy, and so forth;

(iv) subjects without any history of trauma to the maxillofacial area;

(v) subjects who are in a fit state of mind to give a proper history and answer the HADS questionnaire;

(vi) subjects without any previous history of surgery to the maxillofacial area;

(vii) subjects without any history of malignancy who had a past history of malignancy of the maxillofacial area.

Exclusion criteria in patient group include the following:

(i) subjects with history of connective tissue disorders;

(ii) subjects with any comorbid conditions like hypertension, diabetes, asthma, epilepsy, and so forth;

(iii) subjects with any history of trauma to the maxillofacial area;

(iv) subjects who had a history of treatment for TMD in the past;

(v) subjects who were unable to cooperate in the history taking process;

(vi) subjects with any previous history of surgery to the maxillofacial area;

(vii) subjects with any history of malignancy and who had a past history of malignancy of the maxillofacial area.

All the subjects were selected from the outpatient Department of Oral Medicine and Radiology. After explaining the need for study, their consent was obtained on a consent form. A detailed case history was recorded and clinical examination performed by using a structured case history format. The patients with signs and symptoms of TMD were categorized into different groups based on the research diagnostic criteria for TMD (RDC/TMD) [11].

Each subject in group 1 and group 2 completed the hospital anxiety depression scale (HADS) questionnaire.
TABLE 1: Anxiety score in subjects with TMD (males and females).

\begin{tabular}{|c|c|c|c|c|}
\hline \multirow{2}{*}{ Gender } & \multicolumn{3}{|c|}{ Anxiety depression score } & \multirow{2}{*}{ Total } \\
\hline & 0 to 7 & 8 to 10 & 11 to 21 & \\
\hline Male & $81(45.5 \%)$ & $78(43.8 \%)$ & $19(10.6 \%)$ & $178(100 \%)$ \\
\hline Female & $31(40.2 \%)$ & $37(48 \%)$ & $9(11.6 \%)$ & $77(100 \%)$ \\
\hline Total & $112(43.9 \%)$ & $115(45 \%)$ & $28(10.9 \%)$ & $255(100 \%)$ \\
\hline
\end{tabular}

TABLE 2: Anxiety score in subjects without TMD (male and females).

\begin{tabular}{lcccc}
\hline Gender & \multicolumn{5}{c}{ Anxiety score } & Total \\
& 0 to 7 & 8 to 10 & 11 to 21 & \\
\hline Male & $116(78.9 \%)$ & $30(20.4 \%)$ & $1(0.68 \%)$ & $147(100 \%)$ \\
Female & $84(81.5 \%)$ & $18(17.4 \%)$ & $1(0.97 \%)$ & $103(100 \%)$ \\
\hline Total & $200(80 \%)$ & $48(19.2 \%)$ & $2(0.8 \%)$ & $250(100 \%)$ \\
\hline
\end{tabular}

The level of anxiety was rated using the HADS, which consisted of seven items for anxiety. The 7 items for depression in HADS were not considered in this study. By defining the cutoff values, the HADS subscale was used to rate mental disorders as follows [12]:

(i) 0 to 7 being categorized as normal;

(ii) 8 to 10 being categorized as borderline abnormal;

(iii) 11 to 21 being categorized as abnormal.

HADS has been extensively applied and has well-established psychometric properties [13]. The results were tabulated and Fischer's exact test was carried out to analyze the data using IBM SPSS Statistics 19 for Windows (SPSS Inc., Chicago, IL, USA).

\section{Results}

255 subjects presenting with signs and symptoms of TMD were included in group 1 and 250 subjects without any signs and symptoms of TMD were included as controls (group 2). In 255 subjects of group 1, the age ranged from 18 to 49 years with a mean of 29.07 years. In group 2, the age ranged from 19 to 42 years with a mean of 30.12 years. In group 1, there were 178 males and 77 females and, in group 2, there were 147 males and 103 females. The anxiety levels in male and female subjects with TMD and without TMD are shown in Tables 1 and 2, respectively. Within group 1 subjects, there was no statistically significant difference between males and females for the different stress scores (Table 1). The control group also did not show any significant difference between male and female gender (Table 2). Analysis of the results of group 1 and group 2 reveal that the majority of the subjects (both male and female) in group 2 had normal anxiety levels when compared to group 1 subjects (Table 3). Subjects with borderline abnormal anxiety (scores of 8-10) and abnormal anxiety (scores of 11-21) levels were more in group 1 in comparison with group 2 subjects. These results showed a statistically significant difference with regards to anxiety levels between group 1 and group 2 subjects (Tables 3 and 4 ) with $P$ value $<0.0001$. 
TABLE 3: Comparison of anxiety scores in subjects with TMD and control subjects.

\begin{tabular}{lcccccc}
\hline & & \multicolumn{3}{c}{ Anxiety scores } & & \multicolumn{2}{c}{ Females } \\
& & Males & & $0-7$ & $8-10$ & 37 \\
\hline Group 1 & $0-7$ & $8-10$ & $11-21$ & 31 & 9 \\
Group 2 & 116 & 30 & 19 & 84 & 18 & 1 \\
\hline & $P<0.001^{*}$ & $P<0.001^{*}$ & $P<0.0001^{*}$ & $P<0.001^{*}$ & $P<0.001^{*}$ & $P=0.0023^{*}$ \\
\hline
\end{tabular}

*Significant difference for $P$ value in Fischer's exact " $t$ " test.

Group 1: subjects with temporomandibular disorders. Group 2: control subjects.

TABLE 4: Overall anxiety scores between TMD and control subjects.

\begin{tabular}{lcccc}
\hline & \multicolumn{4}{c}{ Anxiety scores } \\
& $0-7$ & $8-10$ & $11-21$ & Total \\
\hline Group 1 & 112 & 115 & 28 & 255 \\
Group 2 & 200 & 48 & 2 & 250 \\
\hline Total & 312 & 163 & 30 & 505 \\
\hline
\end{tabular}

$P<0.0001$.

Group 1: subjects with temporomandibular disorders. Group 2: control subjects.

\section{Discussion}

Being a multifactorial disease affecting the stomatognathic system, TMD is influenced by many factors which includes elevated anxiety levels, symptoms of depression and somatization, and psychological stress. These biopsychosocial factors may be involved in predisposition as well as progression of TMD [14]. The present study reveals significant differences in the anxiety levels between the TMD and control subjects. Greater number of subjects without any signs and symptoms of TMD came under normal anxiety levels on HAD scale in comparison with subjects affected with TMD. Moreover, borderline abnormal and abnormal anxiety scores were significantly more in subjects with TMD in comparison with subjects without signs and symptoms of TMD (Table 3 ). These results concur with several studies which have reported a positive relation between anxiety, depression, and TMD $[7,9,15,16]$. Not only adults but children with high levels of anxiety are found to be 18 times more prone to develop TMD than those without anxiety [17]. Elevated anxiety states in an individual may increase the masticatory muscle activity which in turn may cause TMD [14]. Experimentally induced stress is found to increase masseter activation [18] which may predispose the individual to develop TMD. An HADS score of 13 and above is indicative of significant anxiety and depression [19]. Anxiety and more frequently depression are associated with chronic pain conditions such as TMD $[20,21]$ and it has been estimated that nearly one-third of the patients seeking treatment for TMD suffer from these psychological abnormalities $[22,23]$. One reason for increased anxiety and depression levels in individuals with TMD could be that these patients are emotionally reactive than the healthy normal individuals [8]. It has been proposed that anxiety can be an important predictor of TMD [24] and there is a positive relation between anxiety and TMD. Female gender and middle age group are more prone to develop TMD. The data from the present study shows higher incidence of TMD related signs and symptoms in young male population (Table 1). This difference in the demographics of TMD may be attributed to the occupation of the subjects. Majority of the subjects with signs and symptoms of TMD were working professionals in technical and medical fields. The working environment may play an important role in causation of TMD. It has been postulated that the prevalence of TMD related symptoms is higher among working population due to psychological stress on account of workplace duties, interpersonal relationships, and an achievement oriented climate [25] Depressed mood is one of the significant factor contributing to TMD related symptoms in working population [26]. Physical symptoms such as pain in the head and neck region, eye strain, and chronic back pain can increase due to office environmental factors such as sitting posture and working for long hours on computers [27]. This can alter the psychological profile of the subject $[28,29]$ with increased levels of stress, anxiety, and depression. These changes may predispose the individual to develop TMD. Future studies on etiopathogenesis of TMD should include a thorough evaluation of the psychological profile of the patient. This will help in developing appropriate therapeutic protocols which help in efficient management of these patients.

4.1. Limitations of the Study. Literature review on the prevalence of TMD among the male and female gender reveals that women are more predisposed to TMD when compared to men. However, in our study, this phenomenon is reversed. One reason for this unusual finding could be the gender pattern seeking treatment at our institution. Going through the out patient records of our department, we found more male patients visiting our institution when compared to females. Another reason could be that most of the patients with signs and symptoms of TMD in our study were found to be working in information technology (IT) field. This profession has a slight male predominance. This observation has made us think of examining the role of profession of a patient in the causation of TMD.

\section{Conclusion}

The present study revealed a higher incidence of borderline abnormal and abnormal anxiety scores in TMD subjects in comparison with normal subjects. Multifactorial etiology 
of TMD necessitates the clinician to pay attention to the psychological attributes of the patient. A thorough history emphasizing the psychological aspects of patient's day to day life may reveal significant information regarding the anxiety and stress levels. Unless these factors are taken into consideration, the management strategies for TMD may become inefficient and increase the chances of treatment failure. It is important for the clinician treating TMDs to appreciate the role of anxiety and stress in causation of TMD.

\section{Conflict of Interests}

The authors declare that there is no conflict of interests regarding the publication of this paper.

\section{Acknowledgment}

The authors would like to acknowledge Dr. Seema Patil for her valuable input in preparation of this paper.

\section{References}

[1] M. Ozdemir-Karatas, K. Peker, A. Balik, O. Uysal, and E. B. Tuncer, "Identifying potential predictors of pain-related disability in Turkish patients with chronic temporomandibular disorder pain," The Journal of Headache and Pain, vol. 14, article 17, 9 pages, 2013.

[2] L. Diatchenko, A. D. Anderson, G. D. Slade et al., "Three major haplotypes of the $\beta 2$ adrenergic receptor define psychological profile, blood pressure, and the risk for development of a common musculoskeletal pain disorder," American Journal of Medical Genetics B: Neuropsychiatric Genetics, vol. 141, no. 5, pp. 449-462, 2006.

[3] A. Heiberg, B. Helöe, A. N. Heiberg et al., "Myofascial pain dysfunction (MPD) syndrome in twins," Community Dentistry and Oral Epidemiology, vol. 8, no. 8, pp. 434-436, 1980.

[4] B. S. Michalowicz, B. L. Pihlstrom, J. S. Hodges, and T. J. Bouchard Jr., "No heritability of temporomandibular joint signs and symptoms," Journal of Dental Research, vol. 79, no. 8, pp. 1573$1578,2000$.

[5] A. L. Dougall, C. A. Jimenez, R. A. Haggard, A. W. Stowell, R. R. Riggs, and R. J. Gatchel, "Biopsychosocial factors associated with the subcategories of acute temporomandibular joint disorders," Journal of Orofacial Pain, vol. 26, no. 1, pp. 7-16, 2012.

[6] L. Diatchenko, G. D. Slade, A. G. Nackley et al., "Genetic basis for individual variations in pain perception and the development of a chronic pain condition," Human Molecular Genetics, vol. 14, no. 1, pp. 135-143, 2005.

[7] C. C. Restrepo, L. M. Vásquez, M. Alvarez, and I. Valencia, "Personality traits and temporomandibular disorders in a group of children with bruxing behaviour," Journal of Oral Rehabilitation, vol. 35, no. 8, pp. 585-593, 2008.

[8] L. R. Bonjardim, M. B. Duarte Gavião, L. J. Pereira, and P. M. Castelo, "Anxiety and depression in adolescents and their relationship with signs and symptoms of temporomandibular disorders," International Journal of Prosthodontics, vol. 18, no. 4, pp. 347-352, 2005.

[9] C. L. P. Ferreira, M. A. M. R. da Silva, and C. M. de Felício, "Orofacial myofunctional disorder in subjects with temporomandibular disorder," Cranio, vol. 27, no. 4, pp. 268-274, 2009.
[10] R. A. Pizolato, F. S. F. Fernandes, and M. B. D. Gavião, "Deglutition and temporomandibular disorders in children," Minerva stomatologica, vol. 58, no. 11-12, pp. 567-576, 2009.

[11] S. F. Dworkin and L. LeResche, "Research diagnostic criteria for temporomandibular disorders: review, criteria, examinations and specifications, critique," Journal of Craniomandibular Disorders, vol. 6, no. 4, pp. 301-355, 1992.

[12] N. J. Botega, M. R. Bio, M. A. Zomignani, C. Garcia Jr., and W. A. Pereira, "Mood disorders among inpatients in ambulatory and validation of the anxiety and depression scale HAD," Revista de Saúde Pública, vol. 29, no. 5, pp. 355-363, 1995.

[13] A. Mykletun, E. Stordal, and A. A. Dahl, "The Hospital Anxiety and Depression (HAD) scale: factor structure, item analyses and internal consistency in a large population," British Journal of Psychiatry, vol. 179, no. 1, pp. 540-544, 2001.

[14] I. M. de Lucena, L. L. Franco Rocha Rodrigues, M. L. Teixeira, D. H. Pozza, and A. S. Guimarães, "Prospective study of a group of pre-university students evaluating anxiety and depression relationships with temporomandibular disorders," Journal of Clinical and Experimental Dentistry, vol. 4, no. 2, pp. e102-e106, 2012.

[15] R. B. Fillingim, R. Ohrbach, J. D. Greenspan et al., "Potential psychosocial risk factors for chronic TMD: descriptive data and empirically identified domains from the OPPERA case-control study," Journal of Pain, vol. 12, no. 11, supplement, pp. T46-T60, 2011.

[16] S. Tecco and F. Festa, "Prevalence of signs and symptoms of temporomandibular disorders in children and adolescents with and without crossbites," World Journal of Orthodontics, vol. 11, no. 1, pp. 37-42, 2010.

[17] R. A. Pizolato, F. S. de Freitas-Fernandes, and M. B. D. Gavião, "Anxiety/depression and orofacial myofacial disorders as factors associated with TMD in children," Brazilian Oral Research, vol. 27, no. 2, pp. 156-162, 2013.

[18] C. M. Tsai, S. L. Chou, E. N. Gale, and W. D. Mccall Jr., "Human masticatory muscle activity and jaw position under experimental stress," Journal of Oral Rehabilitation, vol. 29, no. 1, pp. 44-51, 2002.

[19] A. Hinz and E. Brähler, "Normative values for the hospital anxiety and depression scale (hads) in the general German population," Journal of Psychosomatic Research, vol. 71, no. 2, pp. 74-78, 2011.

[20] M. J. Galdon, E. Durá, Y. Andreu, M. Ferrando, R. Poveda, and J. V. Bagán, "Multidimensional approach to the differences between muscular and articular temporomandibular patients: coping, distress, and pain characteristics," Oral Surgery, Oral Medicine, Oral Pathology, Oral Radiology, and Endodontics, vol. 102, no. 1, pp. 40-46, 2006.

[21] R. H. Dworkin and M. J. Gitlin, "Clinical aspects of depression in chronic pain patients," Clinical Journal of Pain, vol. 7, no. 2, pp. 79-94, 1991.

[22] I.-M. Nilsson, "Reliability, validity, incidence and impact of temporormandibular pain disorders in adolescents," Swedish Dental Journal. Supplement, vol. 183, pp. 7-86, 2007.

[23] D. Manfredini, N. Landi, A. Bandettini Di Poggio, L. Dell'Osso, and M. Bosco, "A critical review on the importance of psychological factors in temporomandibular disorders," Minerva stomatologica, vol. 52, no. 6, pp. 321-330, 2003.

[24] V. R. Aggarwal, G. J. Macfarlane, T. M. Farragher, and J. McBeth, "Risk factors for onset of chronic oro-facial pain-results of the North Cheshire oro-facial pain prospective population study," Pain, vol. 149, no. 2, pp. 354-359, 2010. 
[25] M. Sugisaki, N. Takano, K. Kino et al., "Prevalence of temporomandibular disorders among working population of Tokyo," Journal of the Japanese Society for the Temporomandibular Joint, vol. 20, pp. 127-133, 2008.

[26] A. Nishiyama, K. Kino, M. Sugisaki, and K. Tsukagoshi, "Influence of psychosocial factors and habitual behavior in temporomandibular disorder-related symptoms in a working population in Japan," Open Dentistry Journal, vol. 6, no. 1, pp. 240-247, 2012.

[27] T. Nakazawa, Y. Okubo, Y. Suwazono et al., "Association between duration of daily VDT use and subjective symptoms," American Journal of Industrial Medicine, vol. 42, no. 5, pp. 421426, 2002.

[28] Z. Ye, S. Honda, Y. Abe et al., "Influence of work duration or physical symptoms on mental health among Japanese visual display terminal users," Industrial Health, vol. 45, no. 2, pp. $328-$ 333, 2007.

[29] Z. Ye, Y. Abe, Y. Kusano et al., "The influence of visual display terminal use on the physical and mental conditions of administrative staff in Japan," Journal of Physiological Anthropology, vol. 26, no. 2, pp. 69-73, 2007. 


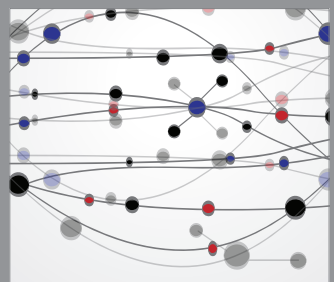

The Scientific World Journal
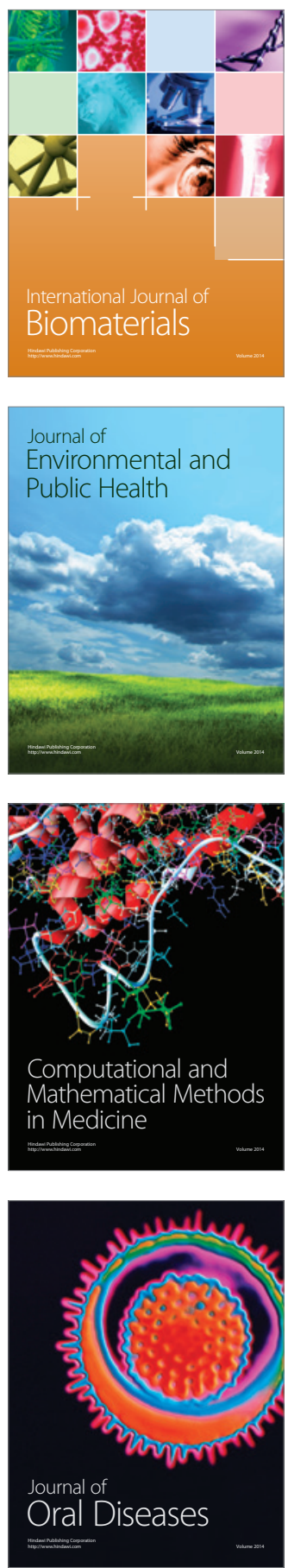
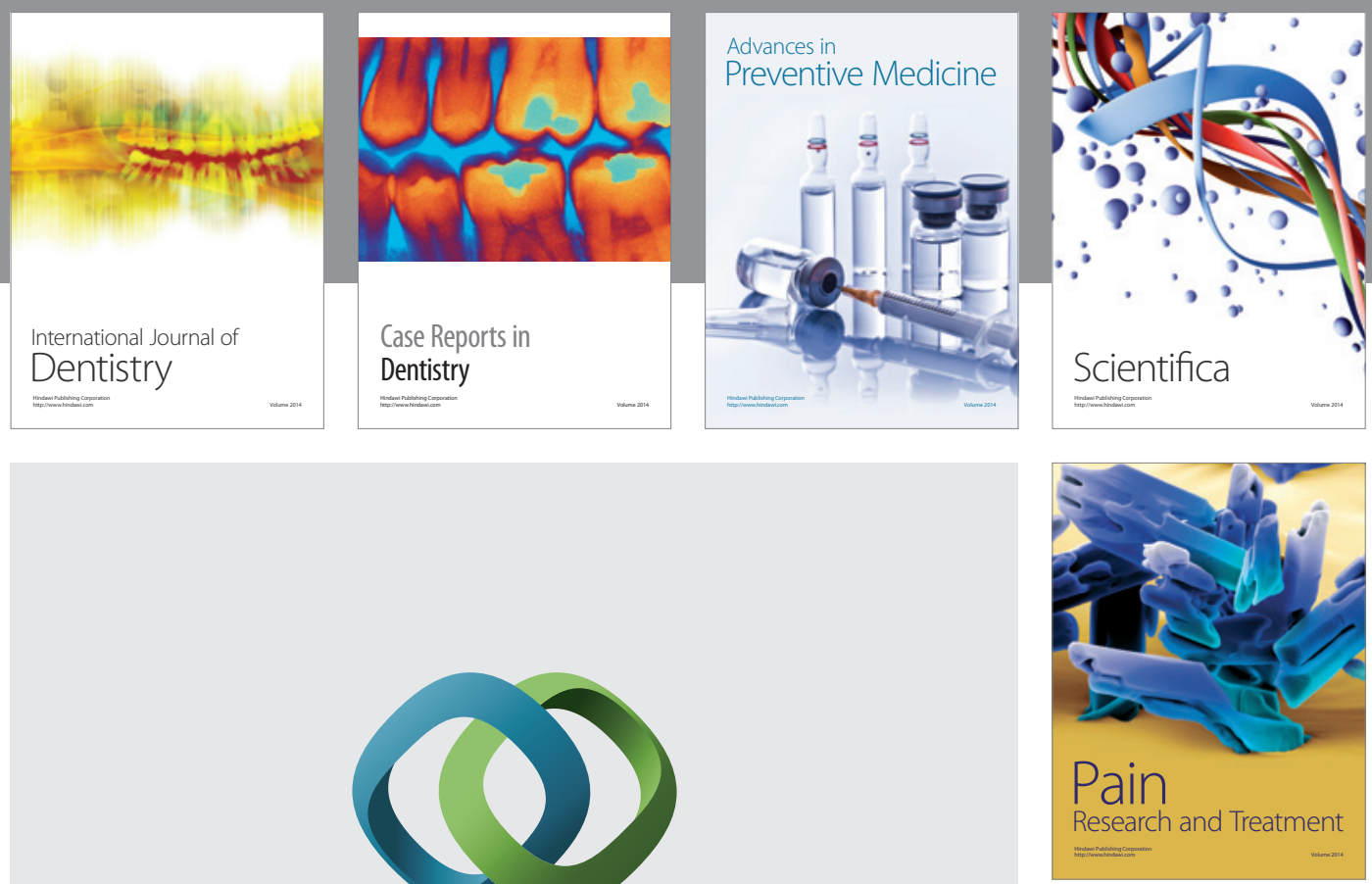

\section{Hindawi}

Submit your manuscripts at

http://www.hindawi.com
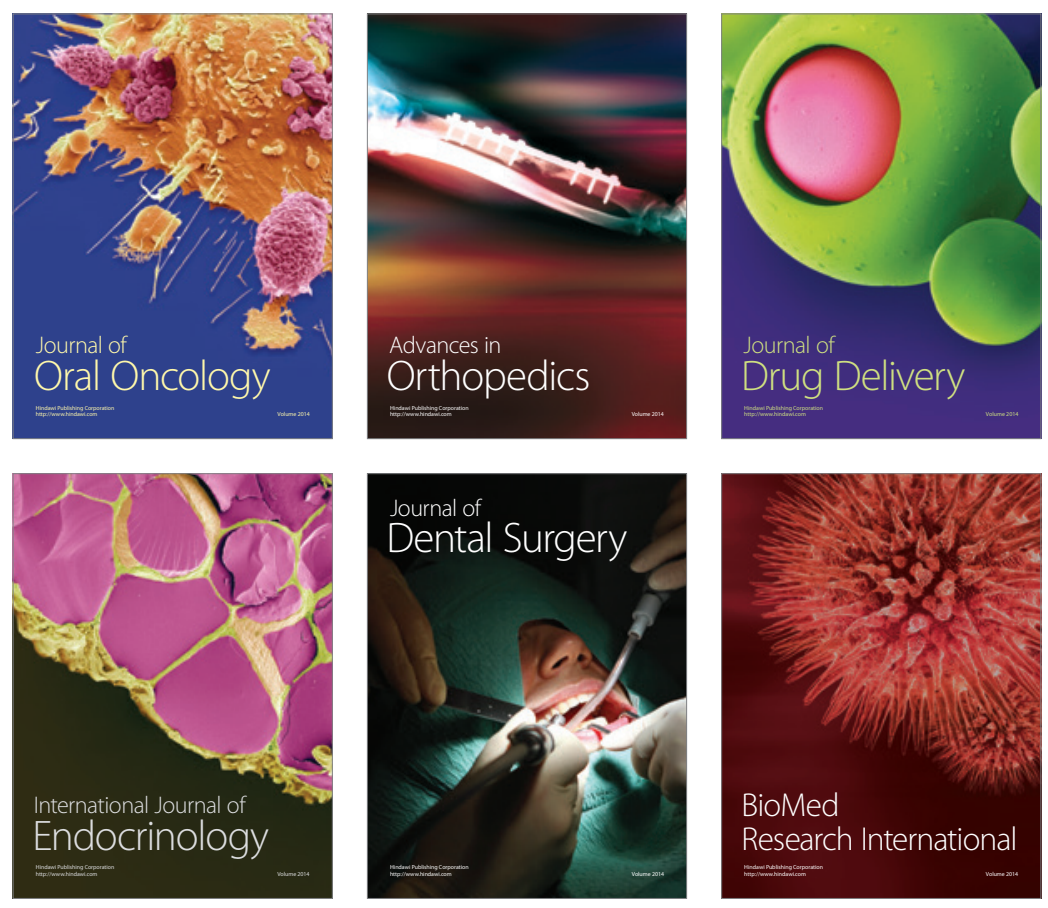

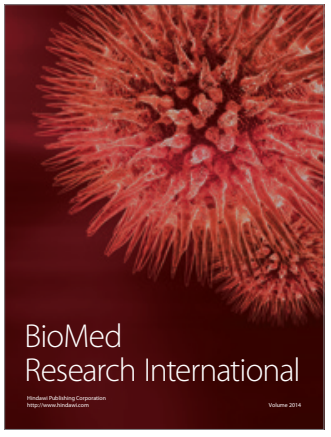

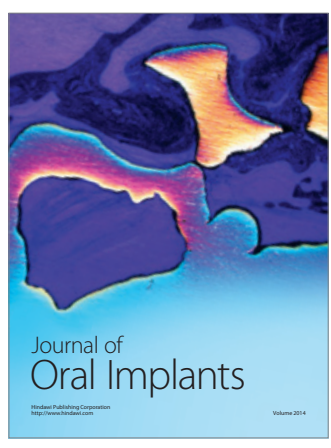
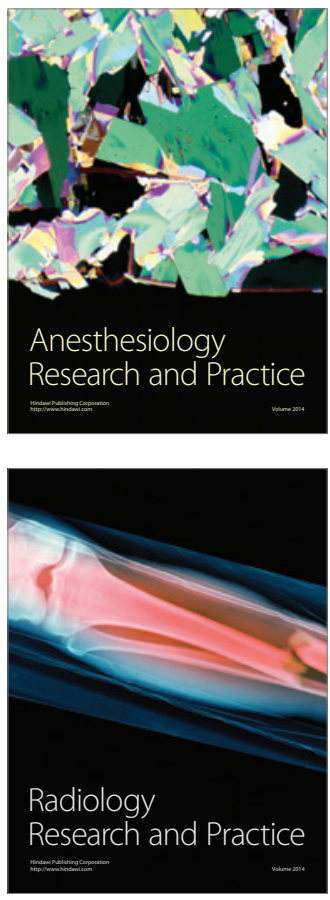